\title{
Failure Analysis of Shaft Circulating Water Pump (CWP) used in Power Plant
}

\author{
Abdul Hamid ${ }^{1, *}$, Sri Nugroho', Gunawan Dwi Haryadi ${ }^{1}$, and Khaeroman ${ }^{2}$ \\ ${ }^{1}$ Department of Mechanical Engineering, Faculty of Engineering, Diponegoro University, \\ Jl. Prof. Soedharto, Tembalang, Semarang 50275 \\ ${ }^{2}$ Department of Mechanical, Politeknik Maritime Negeri Indonesia, Semarang \\ Jl.Pawiyatan Luhur 1/1 Bendan Duwur semarang 50233
}

\begin{abstract}
Pump shafts are generally exposed to the liquid being pumped either on a continual basis or at certain locations along the length of the shaft. The shaft material is austenitic stainless steel, description ASTM AU 79 TY 316. The purpose of this study is to determine the failure of the water pump shaft used in the power plant. Metallography is the study of structure metal shaft can used as a means for CWP metal pelleting (Circulating Water Pump), for the purpose of damaged or deeply degraded areas. SEM test is used to know the beginning of the crack (crack initiation). EDS test is used to chemical composition and Vikers hardness test is also used to know the hardness material. These three tests to support in analyzing the failure of the pump shaft. The conclusion of this failure analysis is the shaft material has porosity. Fatigue cracking comes from the outer surface area.
\end{abstract}

\section{Introduction}

Pump shafts are generally exposed to the liquid being pumped either on a continual basis or at certain locations along the length of the shaft [1]. During operation, the pump shaft usually occurs degradation due to corrosion and or mechanical degradation, usually in the form of fatigue failure. In many cases corrosion precedes fatigue failure and can actually accelerate the rate of failure [1]. Probably the most common cause of failure on pump shafts is fatigue [1].

Fatigue failures on centrifugal shafts are most commonly of the rotating-bending type. Schematic diagrams of the various fractures, which can be expected from rotating bending conditions, are presented in Fig. 1[2]. Fig. 1(a) and (c) illustrate the expected appearance of the fracture surface from a single origin under moderate and high degree of stress concentration while Fig. 1(b) and (d), describes the appearance of fracture surfaces and experiences moderate and high stress concentrations.

Stress concentration sites on shafts where fatigue cracks may initiate are illustrated in Fig. 2 [22]. The most common areas of crack initiation are at the stress concentrations occurring at the keyway root radius and sharp changes in cross-sectional area of the shaft and is referred to as a stress concentration factor [3]. Stress concentrations serve to reduce, the fatigue strength of the component [4].

\footnotetext{
* Corresponding author: hamidsmg80@gmail.com
} 


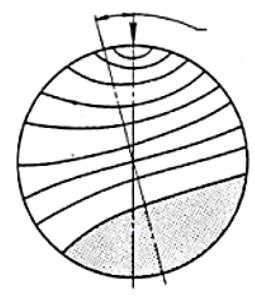

(a)

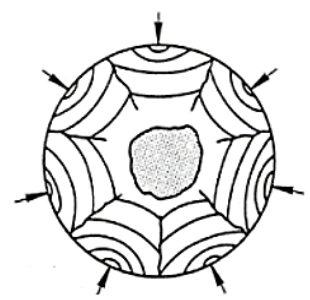

(b)

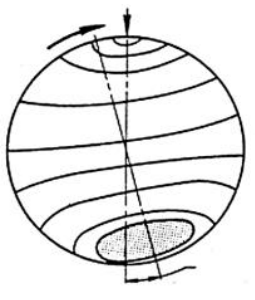

(c)

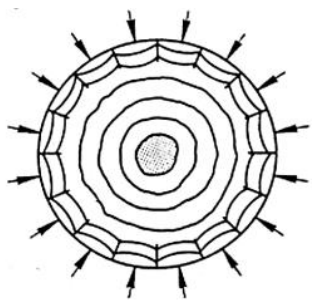

(d)

Fig 1. Schematics of fatigue failure fracture surfaces in rotating bending [2].

Thus, the presence of stress concentrators decreases the allowable stress on the shaft at that point, making it more susceptible to fatigue. Stainless steels on the other hand have an endurance limit ie. The material is design to be use for 8 years but is actually after 12 months usage failure.

By means of visual inspection, it was easy to find that most of the fractures occurred on the weld joints rather than the base materials, material property, manufacturing technology, equipment operation, service environment, routine maintenance or other factors, which were the main causes for inducing these abnormal fractures, were urgently investigated.

Consequently, a comprehensive failure analysis including a variety of characterization methods was conducted to identify the root cause based on our previous failure analysis experiences [5-13].

Actually, many researchers have focused on the properties of decision suport system, such as its fatigue behavior, welding property, corrosion resistance [14-20]. This study investigated the failure analysis of a rotating shaft of a circulating water pump injection, located in an power plan.
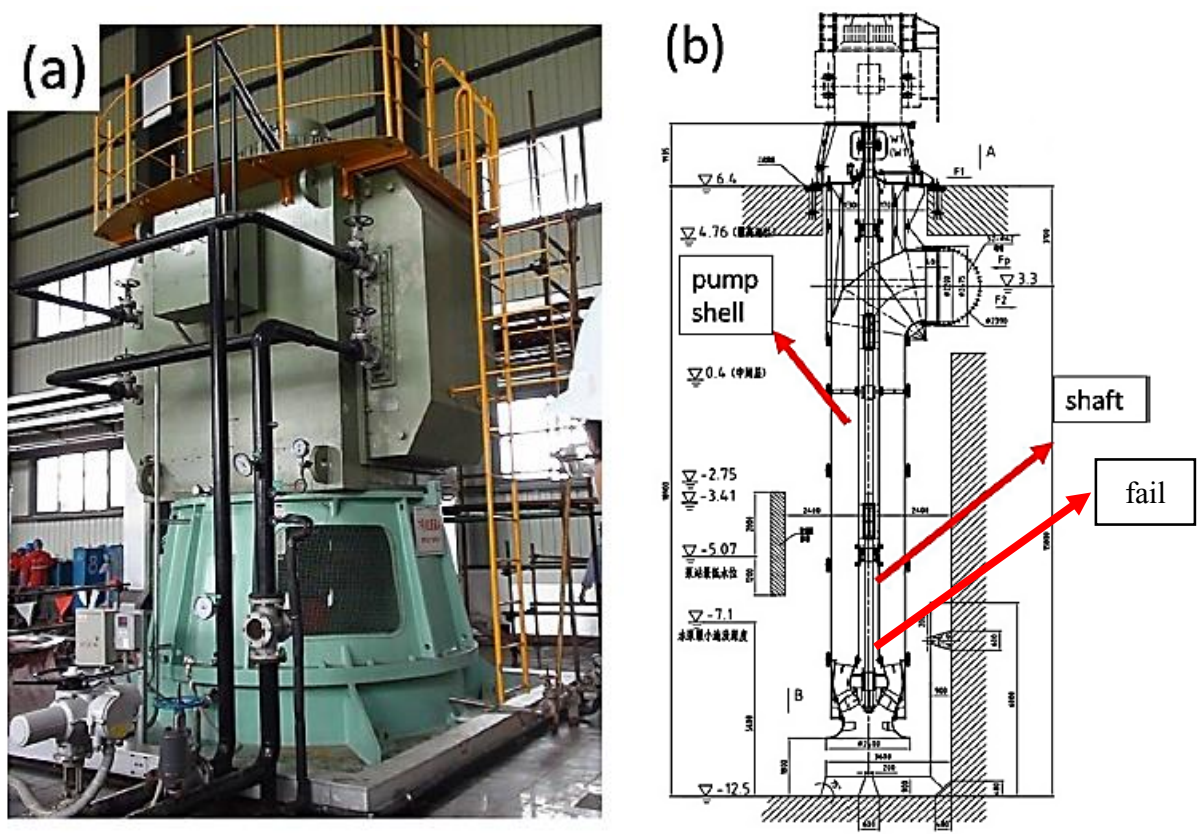

Fig 2. Appearance and structure CWP : (a) Appearance of CWP and (b) Structure of the CWP [22]. 


\section{Materials and methodes}

This rotating shaft failure in service under normal operating conditions of the injection pump during the pumping of brackish water wells for power plan. It is known that water in the operatingn component does not contain a significant amount of hydrogen sulfide $\left(\mathrm{H}_{2} \mathrm{~S}\right)$ generated during the decomposition of seaweed.

The shaft material is an austenitic stainless steel, classification ASTM AU 79 TY 316, supplied in forged condition. In the region of fracture of the shaft. Vickers hardness measurements on the HV were taken in twenty different positions in the cross-section of the shaft in a parallel plane close to the fracture of the part, according to standard.

Metallographic polished surfaces, corresponding to the upper shaft and shaft fracture, were observed with chemical attack in an optical microscope. The microstructure of the material was analyzed via scanning electron microscopy (SEM) to determine its phases.

The microstructure of the shaft material was analysed by optical microscopes. The composition of the shaft material was determined by using a standard spectrometer analyser.

\section{Result and discussion}

\subsection{Visual examination}

Visual examination of the failed end gave the appearance that the shafts of CWP failed by fatigue. A macroscopic view of the failed region of shaft is shown in Fig.3. Signs of smearing and distortion at the key edge were observed.

Areas of damage to the CWP shaft as fatigue failure. Crack propagation begins from an intergranular perlite gap in the yellow arrow. Dynamic layout is displayed on the blue arrow and the red arrow. Static cracking is a very quick propagation in green arrows. The area of damage to the shaft is shown Figure 3.
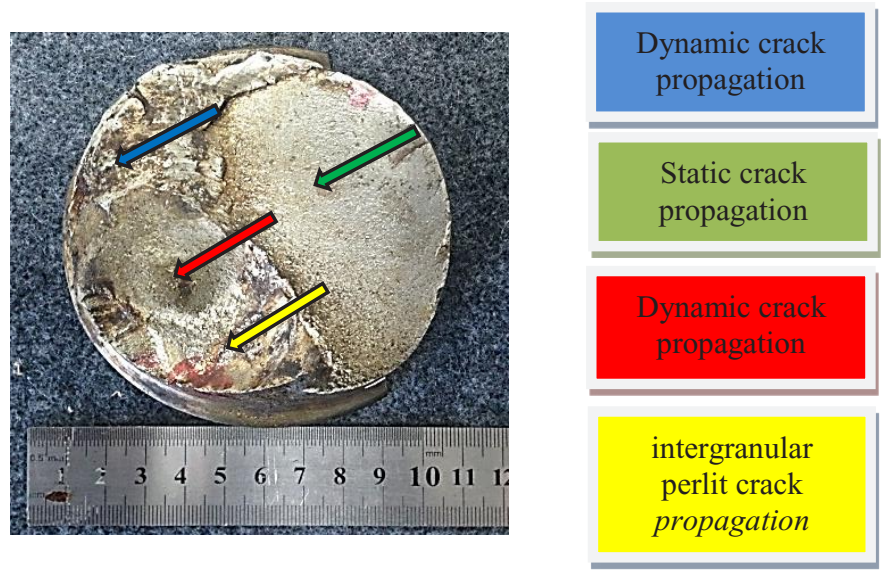

Fig 3. Sites of initiation of fatigue crecks indicated.

\subsection{Chemical composition}

The shaft material is an austenitic stainless steel, classification ASTM AU 79 TY 316 stainless steel enhanced with an addition of $2.5 \%$ Molybdenum, to provide superior corrosion resistance to type 304 stainless steel. 79 TY 316 has improved pitting corrosion resistance and has excellent resistance to sulphates, phosphates and other salts. 79 TY 316 has better resistance than standard 18/8 types to sea water, reducing acids and solution of 
chlorides,bromides and iodies. The material's chemical composition upper shaft $\mathrm{Fe} 67,1 \%$, Cr 17.56\%; Ni 9.97\%; Mo 2.01\%; Mn 1.82\%; Si 0.53\%; and $\mathrm{Cu} 0.49 \%$ and failure shaft are Fe 67\%, Cr 17.45\%; Ni 9.93\%; Mo 2.08\%; Mn 1.82\%; Si 0.55\%; and Cu 0.5\%. From the result of chemical composition test, the value of chemical composition is almost the same on the upper and lower shaft.

\subsection{Hardness test}

Regarding the hardness test, the top axle has a constant HV value and the failure shaft has a very high HV value. Hardness value of the upper shaft and failure shaft are provided in Fig 4 and Fig 5. From the results of hardness testing (HV) obtained a very high abrasion hardness value this proves that the broken shaft fails because of the residual stress on the material. The residual stress causes the axis to be broken suddenly (unexpectedly).

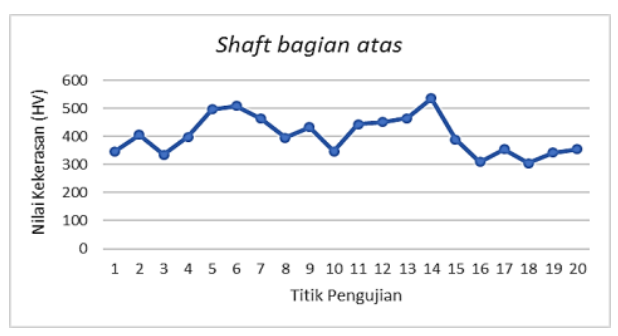

Fig 4. Hardness value upper shaft.

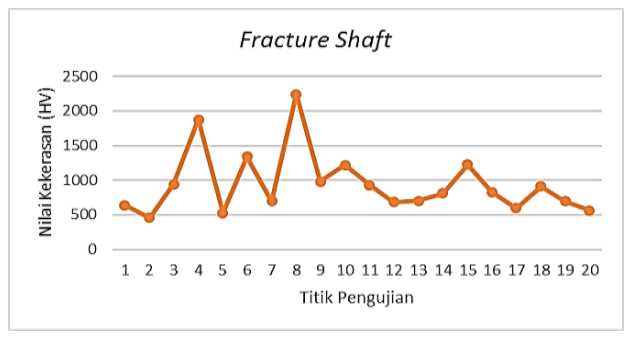

Fig 5. Hardness value failure shaft.

\subsection{Metallography analysis}

Metallographic test can be explained about microstructure type 316 with description description that type 316 is austenitic stainless steel chromiumnickel containing molybdenum. Type 79 TY 316 is an extra-low type 79 TY 316 carbon version that minimizes the precipitation of harmful carbides. With the influence of heat and rapid cooling rate, the pearlite structure is formed.

Figure 6 shows the microstructure of upper shaft and failure shaft. The failure shaft formed has new pearlite, its means that the failure shaft more hardness than the upper shaft.

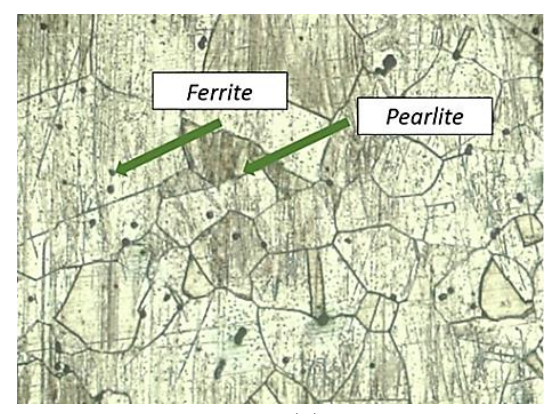

(a).

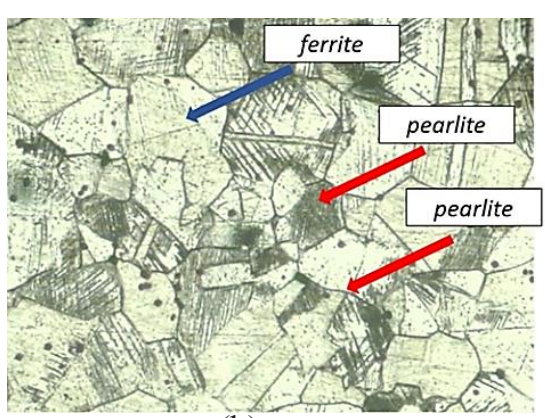

(b).

Fig 6. Microstructure of upper shaft and failure shaft: (a) microstructure of upper shaft and (b) microstructure of failure shaft. 


\subsection{Fractography}

Scanning Electron Microscopy (SEM) is used to fractographic examination. The result of the SEM test we can see the area that became the initial reference of the beginning of the crack. The brittle fracture experienced by the failure shaft can be explained by the contour of a SEM photo marked by a circle, looking very rough because the area has a brittle and high hardness. Figure 7 shows the result SEM in initial crack.

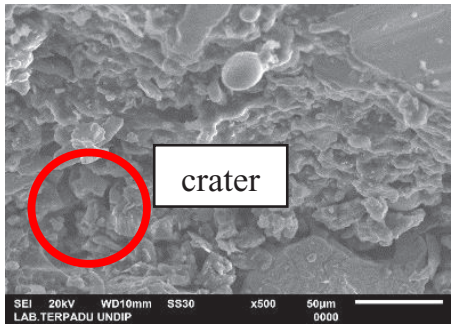

(a)

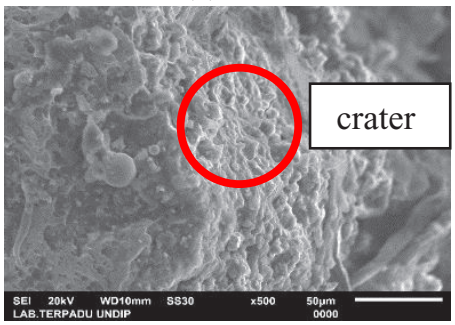

(c)

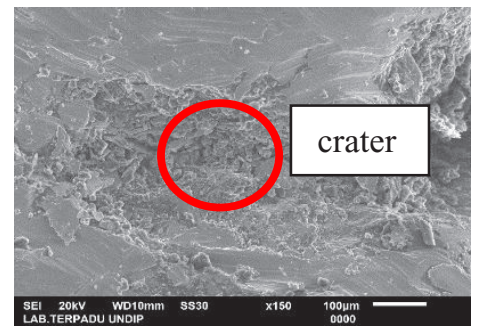

(b)

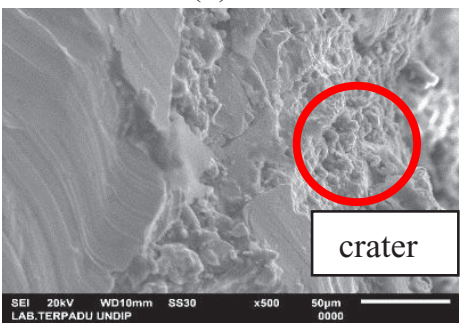

(d)

Fig 7. The result SEM in initial crack (a, b, c, d).

\section{Conclusion}

Chemical composition is almost no change between the shaft failure with a broken shaft. From the composition test, it can be concluded that both shafts include austenite stainless steel type with type AU 79 TY 316. The results of hardness testing on failure shafts tend to be higher than the upper shaft, this may explain that with high hardness levels resulted in rapid crack propagation rates compared with low levels of hardness. On metalography test the influence of heat and the cooling rate are the pearlite structure was formed. With the emergence of the pearlite structure, the properties of the material become hard and brittle. In the SEM test result is the observation reference area is the area experiencing brittle fracture. This can be explained by the contours on the SEM photo looks very rough because the area has a brittle nature and has a high hardness.

The authors gratefully wish to acknowledge the great support provided by Indonesia Power, Reactor Unit in Semarang. The authors extend their sincere thanks to Electrical analysis and engineering measurement reability analysis. Laboratory Management at Center of Research and Services, (UPT. Lab. Terpadu) Diponegoro University, Republic of Indonesia.

\section{References}

1. F. Berndta, A..van Bennekom, Pump shaft failures - a compendium of case studies, Engineering Failure Analysis 8,135 -144. (2001) 
2. ASM Metals Handbook. Failure analysis and prevention, vol. 11. 9th ed. pp. 463. (1985)

3. Peterson RE. S.ress concentration factors. New York: Wiley. (1974)

4. ASM Metals Handbook. Mechanical testing, vol. 8. 9th ed. 364. (1985)

5. Yang ZG, Gong Y, Yuan JZ. Failure analysis of leakage on titanium tubes within heat exchangers in a nuclear power plant. Part I: electrochemical corrosion. Mater Corros;63(1):7-17. (2012)

6. Gong ZG, Yang JZ. Failure analysis of leakage on titanium tubes within heat exchangers in a nuclear power plant. Part II: mechanical degradation. Mater Corros;63(1):18-28. (2012)

7. Gong Y, Yang C, Yao C, Yang ZG. Acidic/caustic alternating corrosion on carbon steel pipes in heat exchanger of ethylene plant. Mater Corros;62(10):967-78. (2010)

8. Gong Y, Yang ZG. Corrosion evaluation of one dry desulfurization equipmentcirculating fluidized bed boiler. Mater Des;32(1):671-81. (2011)

9. Gong Y, Cao J, Ji LN, Yang C, Yao C, Yang ZG. Assessment of creep rupture properties for dissimilar steels welded joints between T92 and HR3C. Fatigue Fract Eng Mater Struct;34(2):83-96. (2011)

10. Gong Y, Yang ZG, Yang FY. Heat strength evaluation and microstructures observation of the welded joints of one China-made T91 steel. J Mater Eng Perform;21(7):1313-9. (2012)

11. Chen Fei-Jun, Yao Chen, Yang Zhen-Guo. Failure analysis on abnormal wall thinning of heat-transfer titanium tubes of condensers in nuclear power plant Part I: corrosion and wear. Eng Fail Anal;37:29-41. (2014)

12. Chen Fei-Jun, Yao Chen, Yang Zhen-Guo. Failure analysis on abnormal wall thinning of heat-transfer titanium tubes of condensers in nuclear power plant Part II: erosion and cavitation corrosion. Eng Fail Anal;37:42-52. (2014)

13. Gong Yi, Yang Zhen-Guo, Meng Xin-Hao.(2013) Failure analysis of one peculiar 'YinYang' corrosion morphology on heat exchanger tubes in purified. terephthalic acid (PTA) dryer. Eng Fail Anal;31:203-10.

14. Iacoviello F, Boniardi M, La Vecchia GM. Fatigue crack propagation in austenoferritic duplex stainless steel 22 Cr $5 \mathrm{Ni}$. Int J Fatigue;21:957-63. (1999)

15. Sieurin H, Sandstrom R. Fracture toughness of a welded duplex stainless steel. Eng Fract Mech;73:377-90. (2006)

16. Sieurin H, Sandstrom R. Sigma phase precipitation in duplex stainless steel 2205. Mater Sci Eng A;444(1-2):271-6. (2007)

17. Lo I-Hsuang, Tsai Wen-Ta. Effect of selective dissolution on fatigue crack initiation in 2205 duplex stainless steel. Corros Sci;49(4):1847-61. (2007)

18. Balbi M, Avalos M, El Bartali A, Alvarez-Armas I. Microcrack growth and fatigue behavior of a duplex stainless steel. Int J Fatigue;31(11-12):2006-13. (2009)

19. Sahu JK, Krupp U, Christ HJ. Fatigue crack initiation behavior in embrittled austeniticferritic stainless steel. Int J Fatigue 2012;45:8-14.

20. Chiu PK, Weng KL, Wang SH, Yang JR, Huang YS, Fang Jason. Low-cycle fatigueinduced martensitic transformation in SAF 2205 duplex stainless steel. Mater Sci Eng A;398(1-2):349-59. (2005) 